\title{
ON MAXIMAL AND MINIMAL QUASISYMMETRIC FUNCTIONS ON AN INTERVAL
}

\author{
MATTI LEHTINEN
}

\section{Introduction}

Let $k \geqq 1$. In recent papers [HH, $\mathrm{H} \mathrm{1,} \mathrm{H} \mathrm{2],} \mathrm{W.} \mathrm{Hayman} \mathrm{and} \mathrm{A.} \mathrm{Hinkkanen}$ have investigated the maximal distortion under a normalized $k$-quasisymmetric function $f$ of the real line. They denote the family of all such functions, normalized by $f(-1)=-1$ and $f(1)=1$, by $N_{0}(k)$. Hayman and Hinkkanen have found bounds for the growth of $f \in N_{0}(k)$, and Hinkkanen has constructed piecewise linear functions $f_{0} \in N_{0}(k)$ for which $f_{0}(x)=\max \left\{f(x) \mid f \in N_{0}(k)\right\}$ for infinitely many $x$.

In this note, we consider the related problem of the distortion under a $k$-quasisymmetric self-map of $[0,1]$, i.e., under a function $f:[0,1] \rightarrow[0,1]$ satisfying $f(0)=0, f(1)=1$, and

$$
(f(x)-f(x-t)) / k \leqq f(x+t)-f(x) \leqq k(f(x)-f(x-t))
$$

for all $x$ and $t$ such that $[x-t, x+t] \subset[0,1]$. We denote the family of all such functions by QS $(k)$. In particular, we investigate whether the functions introduced by R. Salem and studied by K. Goldberg [S, G] are best possible majorants or minorants of the functions in QS $(k)$. In the last section, we apply our results to the dilatation estimates of the Beurling-Ahlfors extensions. - In a sense, the distortion problem is a one-dimensional analogue of the problem of distortion under a $K$-quasiconformal self-map of the unit disc.

If $f \in N_{0}(k)$, then $g$, defined by $g(x)=(f(2 x-1)+1) / 2$ for $x \in[0,1]$, is in QS $(k)$. All functions in QS $(k)$ are not restrictions of $k$-quasisymmetric functions of the real line. (See the remark after Proposition 3.) On the other hand, boundedness of the domain and range of the mappings rules out a behaviour which condition (1) allows for mappings of the real line:

Proposition 1. If $f \in \mathrm{QS}(k)$, then $f$ is a homeomorphism.

Proof. If $k>1$, a subtraction of the right and left hand sides of (1) shows that $f$ is nondecreasing. It is easy to see that in this case $f$ is continuous: let $c \in(0,1)$ and assume $\lim _{x \rightarrow c^{-}} f(x)=a<b=\lim _{x \rightarrow c^{+}} f(x)$. Given an $\varepsilon<(b-a) / k$, there is a $\delta$ such that $f(x)<b+\varepsilon$ for $c<x<c+\delta$. Choose $x$ and $t$ so that $x-t<c<x<$ 
$x+t<c+\delta$ to obtain a contradiction with (1). Similarly one can see that $f$ is continuous at 0 and 1 . Consequently, $f$ is a homeomorphism.

This argument does not work if $k=1$ (cf. [G]; in fact, functions satisfying (1) but defined on an unbounded interval need not be monotonic). However, assuming $k=1$ and, say, $f(x)=a>x$ for an $x>1 / 2$, we obtain from (1) that for some $n$ we have $0<y=2^{n} x-2^{n}+1<1 / 2$ and $f(y)=2^{n} a-2^{n}+1>y$. Since $f\left(y+p 2^{-q}(x-y)\right)=f(y)+$ $p 2^{-q}(f(x)-f(y))$ for all integers $q \geqq 1$ and $0 \leqq p \leqq 2^{q}$, there is a $z<1 / 2$ such that $f(z)>1 / 2$. But then $f(2 z)>1$, which is not possible. So, if $k=1$, then $f(x)=x$ for all $x$.

Beurling and Ahlfors [BA] were the first to ask for $M_{k}(x)=\max \{f(x) \mid f \in \mathrm{QS}(k)\}$ and $m_{k}(x)=\min \{f(x) \mid f \in \mathrm{QS}(k)\}$, since the computations needed for the estimation of the maximal dilatation of their extension of a quasisymmetric function to a quasiconformal self-map of the half-plane depended on upper and lower bounds of the integral

$$
I=\int_{0}^{1} f(x) d x
$$

for $f \in \mathrm{QS}(k)$. However, the needed inequalities $1 /(1+k) \leqq I \leqq k /(1+k)$ were easy to establish without direct knowledge of $m_{k}$ or $M_{k}$ ([BA, p. 137]; see [Le] for a slightly sharpened version).

Because $f$ and $g$, where $g(x)=1-f(1-x)$, either both are in QS $(k)$ or are not in QS $(k), m_{k}(x)=1-M_{k}(1-x)$ for all $x \in[0,1]$.

\section{Salem's functions}

K. Goldberg [G] observed the connection between $m_{k}, M_{k}$ and the completely singular homeomorphisms of $[0,1]$ introduced by $\mathrm{R}$. Salem [S]. Setting $\lambda=\lambda(k)=$ $1 /(1+k), \mu=\mu(k)=k \lambda$, we define the upper Salem function $P=P_{k}$ inductively for points with a finite dyadic representation by $P(0)=0, P(1)=1$, and by

$$
P\left((2 j+1) / 2^{n}\right)=\lambda P\left(j / 2^{n-1}\right)+\mu P\left((j+1) / 2^{n-1}\right)
$$

for $n=1,2, \ldots ; j=0,1, \ldots, 2^{n-1}-1$, and for the rest of $[0,1]$ by continuity. The lower Salem function $p_{k}$ is similarly defined by exchanging $\lambda$ and $\mu$ in (2), and $p_{k}(x)=$ $1-P_{k}(1-x)$ for all $x$ [G]. Since it follows easily from (1) that every $f \in \mathrm{QS}(k)$ satisfies

$$
\mu f(a)+\lambda f(b) \leqq f((a+b) / 2) \leqq \lambda f(a)+\mu f(b)
$$

for $0 \leqq a<b \leqq 1$, we see that

$$
p_{k}(x) \leqq m_{k}(x), \quad M_{k}(x) \leqq P_{k}(x)
$$


for all $x \in[0,1]$. The examples

$$
\begin{aligned}
& f(x)= \begin{cases}4 \mu^{2} x, & x \in[0,1 / 4] \\
\mu^{2}+4 \mu \lambda(x-1 / 4), & x \in[1 / 4,3 / 4] \\
\mu+\mu \lambda+4 \lambda^{2}(x-3 / 4), & x \in[3 / 4,1],\end{cases} \\
& g(x)= \begin{cases}4 \lambda^{2} x, & x \in[0,1 / 4] \\
\lambda^{2}+4 \mu \lambda(x-1 / 4), & x \in[1 / 4,3 / 4] \\
\lambda+\mu \lambda+4 \mu^{2}(x-3 / 4), & x \in[3 / 4,1],\end{cases}
\end{aligned}
$$

show that there are points $x$ for which $M_{k}(x)=P_{k}(x)$ or $m_{k}(x)=p_{k}(x)$ (in the examples, $x=j / 4, j=0,1,2,3,4)$. On the other hand, if $k>1$, then $P=P_{k} \notin \mathrm{QS}\left(k^{\prime}\right)$ for any $k^{\prime}$, since

$$
\left(P\left(1 / 2+1 / 2^{n}\right)-P(1 / 2)\right) /\left(P(1 / 2)-P\left(1 / 2-1 / 2^{n}\right)\right)=k^{n-2}
$$

for $n \geqq 1$.

A direct consequence of Goldberg's observation is

Proposition 2. $M_{k}$ and $m_{k}$ are continuous and strictly increasing.

Proof. Only the statements concerning $M_{k}$ have to be proved. Clearly, $M_{k}$ is non-decreasing. Assume, for instance, that $\lim _{x \rightarrow c^{+}} M_{k}(x)>M_{k}(c)$. Then there exist a $\delta>0$, and sequences $\left(x_{j}\right), x_{j}>c, \lim _{j \rightarrow \infty} x_{j}=c$, and $\left(f_{j}\right), f_{j} \in \mathrm{QS}(k)$ such that $f_{j}\left(x_{j}\right)>$ $M_{k}(c)+\delta$. Set $g_{j}(t)=f_{j}\left(t x_{j}\right) / f_{j}\left(x_{j}\right)$. Then $g_{j} \in \mathrm{QS}(k)$, and $p_{k}\left(c / x_{j}\right) \leqq g_{j}\left(c / x_{j}\right)=$ $f_{j}(c) / f_{j}\left(x_{j}\right) \leqq M_{k}(c) /\left(M_{k}(c)+\delta\right)<1$, in contradiction with $\lim _{j \rightarrow \infty} p_{k}\left(c / x_{j}\right)=1$.

Similarly, if $M_{k}(a)=M_{k}(b), a<b$, we find $\left(f_{j}\right), f_{j} \in \mathrm{QS}(k)$, such that $f_{j}(a)>$ $M_{k}(a)-1 / j$. Define $g_{j} \in \mathrm{QS}(k)$ by $g_{j}(x)=\left(f_{j}(x+(1-x) a)-f_{j}(a)\right) /\left(1-f_{j}(a)\right)$. Then $g_{j}((b-a) /(1-a)) \rightarrow 0$, in contradiction with (4) and the fact that $p_{k}(c)>0$ for $c>0$.

\section{Piecewise linear quasisymmetric functions}

We shall investigate the possibility of equality in (4); our previous example shows that equality is true for certain values of $x$ at least.

An obvious device for proving an equality $M_{k}(x)=y$ is to construct a piecewise linear function $f \in \mathrm{QS}(k)$ satisfying $f(x)=y$.

The following lemma, which is proved in [HH, p. 64], facilitates the proof that a given piecewise linear function is in QS $(k)$ :

Lemma 1. Let $S \subset[0,1],\{0,1\} \subset S$, be a discrete set and $f:[0,1] \rightarrow[0,1]$, $f(0)=0, f(1)=1$, be continuous on $[0,1]$ and linear on each interval in $[0,1] \backslash S$. Then $f \in \mathrm{QS}(k)$ if and only if (1) is true for all $x, t$ such that $\{x-t, x, x+t\} \cap S$ has at least two elements. 
Remark. Even if $S$ is finite, the number of relevant intersections to be checked grows with the cardinality of $S$ : in fact, if $S$ has $n$ elements, then there are at least $n^{2} / 2-3 n / 2+1$ and at most $3 n^{2} / 2-11 n / 2+5$ intersections to be checked.

As a corollary to Lemma 1 , we obtain

Lemma 2. Let $f \in \mathrm{QS}(k)$ be piecewise linear and $f(1 / 2)=\mu$.

(a) If $f$ is linear on $[1 / 2,1]$, and $g(x)=\mu f(2 x)$ for $x \in[0,1 / 2], g(x)=f(x)$ for $x \in[1 / 2,1]$, then $g \in \mathrm{QS}(k)$.

(b) If $f$ is linear on $[0,1 / 2]$, and $g(x)=f(x)$ for $x \in[0,1 / 2], g(x)=\mu+\lambda f(2 x-1)$ for $x \in[1 / 2,1]$, then $g \in \mathrm{QS}(k)$.

Proof. (a) For any homeomorphism $h$, we denote the quotient

$$
(h(x+t)-h(x)) /(h(x)-h(x-t))
$$

by $q_{n}$. Denote by $S_{f}\left(S_{g}\right)$ the set of points where $f(g)$ is not linear. Choose $x, t$ such that two of the points $x-t, x, x+t$ are in $S_{g}$. Then two of the points $2 x-2 t$, $2 x, 2 x+2 t$ are in $S_{f}$ and $q_{g}(x, t)=q_{f}(2 x, 2 t)$, except when $x+t=1$. In this case $x=t=1 / 2$ and $q_{g}(x, t)=1 / k$. The proof for $(\mathrm{b})$ is similar.

If we apply Lemma 2 repeatedly to $f, f(x)=2 \mu x, x \in[0,1 / 2], f(x)=\mu+2 \lambda(x-1 / 2)$, $x \in[1 / 2,1]$, and observe that $x \rightarrow 1-f(1-x)$ is in QS $(k)$ together with $f$, we immediately get infinitely many points $x$ at which $M_{k}(x)=P_{k}(x)$ :

Proposition 3. For every natural number $n, \quad M_{k}\left(1 / 2^{n}\right)=\mu^{n}=P_{k}\left(1 / 2^{n}\right)$,

$$
\begin{gathered}
M_{k}\left(1-1 / 2^{n}\right)=1-\lambda^{n}=P_{k}\left(1-1 / 2^{n}\right) ; m_{k}\left(1 / 2^{n}\right)=p_{k}\left(1 / 2^{n}\right)=\lambda^{n}, \\
m_{k}\left(1-1 / 2^{n}\right)=p_{k}\left(1-1 / 2^{n}\right)=1-\mu^{n} .
\end{gathered}
$$

Remark. The construction above gives an example of functions $f \in Q S(k)$ with no $k$-quasisymmetric extension to the real line. Indeed, if $f\left(1 / 2^{n}\right)=\mu^{n}, f(1 / 2)=\mu$ and $f$ is linear on $[1 / 2,1]$, then a $k$-quasisymmetric extension of $f$ would have

$$
f\left(-1 / 2^{n}\right) \geqq-\mu^{n-1} \lambda, \quad f\left(1+1 / 2^{n}\right) \leqq 1+\mu / 2^{n},
$$

and $q_{f}\left(1 / 2,1 / 2+1 / 2^{n}\right) \leqq(1 / k)\left(1+\mu /\left(\lambda 2^{n}\right)\right) /\left(1+\mu^{n-2} \lambda\right)<1 / k$ for $n$ large enough. (Since $\mu>1 / 2$, the numerator tends to 0 faster than the denominator.) - Of course, every $f \in \mathrm{QS}(k)$ has a $k_{1}$-quasisymmetric extension to $\mathbf{R}$, with $k_{1}>k$ depending on $k$ only.

Further evidence supporting the hypothesis $P_{k}=M_{k}$ is obtained from the piecewise linear functions which are linear on intervals in $[0,1] \backslash S$, $S=\{j / 8 \mid j=0,1, \ldots, 8\}$, and agree with $P_{k}$ or $p_{k}$ on $S$. A direct check gives

Proposition 4. For $j=0,1, \ldots, 8, M_{k}(j / 8)=P_{k}(j / 8), m_{k}(j / 8)=p_{k}(j / 8)$. 


\section{The points $1 / 3$ and $2 / 3$}

From (3), we obtain for $f \in \mathrm{QS}(k)$

Solving, we get

$$
\begin{gathered}
\mu f(1 / 3)+\lambda \leqq f(2 / 3) \leqq \lambda f(1 / 3)+\mu, \\
\lambda f(2 / 3) \leqq f(1 / 3) \leqq \mu f(2 / 3) .
\end{gathered}
$$

$$
\begin{gathered}
\lambda^{2} /(1-\mu \lambda) \leqq f(1 / 3) \leqq \mu^{2} /(1-\mu \lambda), \\
\lambda /(1-\mu \lambda) \leqq f(2 / 3) \leqq \mu /(1-\mu \lambda) .
\end{gathered}
$$

Using (2) and the representation

we obtain

$$
1 / 3=1 / 4+\sum_{n=2}^{\infty}\left(1 / 2^{2 n-1}-1 / 2^{2 n}\right),
$$

$$
P_{k}(1 / 3)=\mu^{2} \sum_{n=0}^{\infty}(\mu \lambda)^{n}=\mu^{2} /(1-\mu \lambda) .
$$

Similarly, the other three upper and lower bounds in (5) and (6) are the respective values of the upper and lower Salem functions.

However, the equality $M_{k}(1 / 3)=P_{k}(1 / 3)$ does not hold. In fact, setting $f(j / 12)=a_{j}$ for brevity, we obtain from (3) that $a_{4} \leqq \mu a_{8}, a_{8} \leqq \lambda a_{7}+\mu a_{9}$ or $a_{4} / \mu-$ $\mu a_{9} \leqq \lambda a_{7}$ and $a_{4} \leqq \lambda a_{3}+\mu a_{5}$. Since $\mu a_{5}+\lambda a_{7} \leqq a_{6}$ we get

$$
a_{4}-\lambda a_{3}+a_{4} / \mu-\mu a_{9} \leqq a_{6} .
$$

Inserting $a_{3} \leqq M_{k}(1 / 4)=\mu^{2}, a_{6} \leqq M_{k}(1 / 2)=\mu, a_{9} \leqq M_{k}(3 / 4)=\mu+\mu \lambda$ we obtain

$$
f(1 / 3)=a_{4} \leqq \mu^{2}(1+\mu+2 \mu \lambda) /(1+\mu) .
$$

It is easy to check that the right hand side in (7) is indeed strictly less than $P_{k}(1 / 3)$ as soon as $k>1$.

A similar argument improves the right hand side of (6) to

$$
f(2 / 3) \leqq \mu\left(1+\lambda+\lambda \mu+2 \mu \lambda^{2}\right) /(1+\lambda)<P_{k}(2 / 3) .
$$

Denote the upper bounds in (T) and (8) by $\varkappa=\varkappa(k)$ and $v=v(k)$, respectively. Using the fact that $g, g(x)=1-f(1-x)$, belongs to QS $(k)$ together with $f$, we get the lower bounds $1-v \leqq f(1 / 3), 1-x \leqq f(2 / 3)$.

We observe the strict inequalities $x<\mu v$ and $v<\lambda x+\mu$. These imply that the simultaneous equations $q_{f}(x, t)=k$ and $q_{f}(x+t, t)=k$ or $q_{f}(x, t)=1 / k$ and $q_{f}(x+t, t)=1 / k$ cannot hold for any $k$-quasisymmetric function.

To prove that $M_{k}(1 / 3)=\varkappa, M_{k}(2 / 3)=v$, we construct piecewise linear functions $f, g \in \mathrm{QS}(k)$ such that $f(1 / 3)=x, g(2 / 3)=v$.

Proposition 5. $M_{k}(1 / 3)=1-m_{k}(2 / 3)=\varkappa, M_{k}(2 / 3)=1-m_{k}(1 / 3)=v$.

Proof. In view of the restrictions described above, any $f \in \mathrm{QS}(k)$ satisfying $f(1 / 3)=\varkappa$ must also satisfy $f(1 / 4)=\mu^{2}, f(1 / 2)=\mu, f(3 / 4)=\mu+\mu \lambda, f(2 / 3) \geqq x / \mu$, 
$f(5 / 12)=x / \mu-\mu \lambda$, and $f(7 / 12)=\left(x / \mu-\mu^{2}\right) / \lambda-\mu^{2}$. In addition, (1) requires $f(5 / 6) \geqq$ $f(5 / 12) / \mu$. Finally, (1) with $x=1 / 3, t=1 / 6$ requires $f(1 / 6) \geqq x-k(\mu-x)$, and with $x=1 / 3, t=1 / 4$ requires $f(1 / 12) \geqq(1+k) x-(k / \lambda)\left(x / \mu-\mu^{2}(1+\lambda)\right)$. Define a piecewise linear function $f$, linear in $[0,1] \backslash S, S=\{0,1 / 12, \ldots, 10 / 12,1\}$ by replacing all the inequality signs in the above conditions by equalities; a check by Lemma 1 shows that $f$ is in QS $(k)$. Similarly, a piecewise linear $g$ satisfying $g(2 / 3)=v$, $g(1 / 6)=\mu^{2} /(1+\lambda), \quad g(1 / 4)=\mu^{2}, \quad g(1 / 3)=\mu \nu, \quad g(5 / 12)=\mu-\mu \lambda^{2} /(1+\lambda), \quad g(1 / 2)=\mu$, $g(7 / 12)=\mu+\mu^{2} \lambda /(1+\lambda), g(3 / 4)=\mu(1+\lambda), g(5 / 6)=\mu+\mu \lambda(1+2 \lambda) /(1+\lambda), g(11 / 12)=$ $\left(1+\mu \lambda+\mu \lambda^{2}\right) /(1+\lambda)$ is in QS $(k)$.

Corollary. If $k>1$, the set of $x$ for which $M_{k}(x)=P_{k}(x)$ is nowhere dense, and $M_{k}(x)<P_{k}(x)$ for infinitely many $x$ with a finite dyadic representation.

Proof. If $M_{k}(x)=P_{k}(x)$ on an interval, then $M_{k}(x)=P_{k}(x)$ on an interval whose endpoints are $m / 2^{n}$ and $(m+1) / 2^{n}$. But $M_{k}\left((m+1 / 3) / 2^{n}\right)<P_{k}\left((m+1 / 3) / 2^{n}\right)$.

Propositions 2 and 5 as well as the continuity of $P_{k}$ also imply that there are intervals on which $M_{k}<P_{k}$. Majorants of $M_{k}$, sharper than $P_{k}$, can be constructed in a way similar to the construction of Salem's function but using both (3) and the inequalities $f(2 a / 3+b / 3) \leqq(1-x) f(a)+x f(b), f(a / 3+2 b / 3) \leqq(1-v) f(a)+v f(b) \quad$ on subintervals of $[0,1]$.

By using Proposition 5, we can also easily prove

Proposition 6. If $k>1, M_{k}$ is not in QS $(k)$.

Proof. We show that no $f$ which satisfies $f(1 / 3)=x$ and $f(2 / 3)=v$ is in QS $(k)$. Assuming the contrary, we get $x \leqq \lambda f(1 / 4)+\mu f(5 / 12), v \leqq \lambda f(7 / 12)+\mu f(3 / 4)$ and hence $x+\nu \leqq \mu^{2} \lambda+\mu^{2}(1+\lambda)+\mu f(5 / 12)+\lambda f(7 / 12) \leqq \mu+2 \mu^{2} \lambda+\mu^{2}$. Inserting the formulas for $\varkappa$ and $\nu$ and simplifying, we get the contradiction $\mu \leqq 1 / 2$.

\section{On the dilatation of the Beurling-Ahlfors extension}

The Beurling-Ahlfors extension $F$ of $f \in \mathrm{QS}(k)$ is defined in

$$
T=\{z=x+i y \mid 0 \leqq x \leqq 1,0<y \leqq \min \{x, 1-x\}\}
$$

by $2 F(z)=\alpha_{0}(z)+\alpha_{1}(z)+\operatorname{ir}\left(\alpha_{0}(z)-\alpha_{1}(z)\right)$ where

$$
\alpha_{j}(z)=\int_{0}^{1} f\left(x+(-1)^{j} y t\right) d t, \quad j=0,1, \quad z=x+i y,
$$

and $r>0$. By [BA] and [Le], $r$ can be chosen so that the maximal dilatation $K_{F}$ of $F$ is at most $\min \left\{k^{3 / 2}, 2 k-1\right\}$. On the other hand, for $k>12$ there are examples of $k$-quasisymmetric functions $f$ defined on the whole real line for which every Beurling-Ahlfors extension has maximal dilatation at least $3 k / 2$ [Le] and for large $k$ even larger than $1.587 k$ [Li]. 
Restricting ourselves to $k>7$, we find functions $f \in \mathrm{QS}(k)$ for which $K_{F}>8 k / 5$ for every $r$. To this end, let $f(j / 8)=P_{k}(j / 8), j=0, \ldots, 8$, and let $f$ be linear on each interval $[j / 8,(j+1) / 8]$ (cf. Proposition 4). Passing to $g, g(x)=1-f((1-x) / 2) / \mu$, and computing the dilatation $D$ of the Beurling-Ahlfors extension of $g$ at the point $i$ as in [Le, p. 139], we arrive at $D>8 k / 5$ for $k>7$ and $\lim _{k \rightarrow \infty} D / k=1+$ $49 / 64=1.765625$. Observe, however, that $f$ has no $k$-quasisymmetric extension to the real line. Such an extension ought to satisfy $f(9 / 8) \leqq 1+\mu \lambda^{2}$ and also $f(5 / 4) \geqq$ $f(5 / 8) / \mu=1+\mu \lambda$. Since $f(7 / 8)=1-\lambda^{3}$, this would imply that $f(5 / 4)-f(9 / 8)=$ $k(f(9 / 8)-f(1))=k^{2}(f(1)-f(7 / 8))$, which clearly contradicts the remark preceding Proposition 5.

\section{References}

[BA] Beurling, A., and L. V. Ahlfors: The boundary correspondence under quasiconformal mappings. - Acta Math. 96, 1956, 125-142.

[G] Goldberg, K.: A new definition for quasisymmetric functions. - Michigan Math. J. 21, $1974,49-62$.

[HH] Hayman, W., and A. Hinkkanen: Distortion estimates for quasisymmetric functions. Ann. Univ. Mariae Curie-Skłodowska Sect. A 36-37, 1982-83, 51-67.

[H 1] Hinkkanen, A.: Quasisymmetric functions of extremal growth. - Ann. Acad. Sci. Fenn. Ser. A I Math. 11, 1986, 63-75.

[H 2] Hinkkanen, A.: Asymptotic extremal growth of quasisymmetric functions. - Ann. Acad. Sci. Fenn. Ser. A I Math. 11, 1986, 295-319.

[Le] Lehtinen, M.: Remarks on the maximal dilatation of the Beurling-Ahlfors extension. Ann. Acad. Sci. Fenn. Ser. A I Math. 9, 1984, 133-139.

[Li] Lr ZHONG: On the Beurling-Ahlfors extension. - Acta Math. Sinica 26, 1983, 279-290 (Chinese).

[S] SALEM, R.: On some singular monotonic functions which are strictly increasing. - Trans. Amer. Math. Soc. 53, 1943, 427-439.

University of Helsinki

Department of Mathematics

SF-00100 Helsinki

Finland

Received 5 February 1986 